\title{
Expression and correlation of HSP70, Apaf-1 and ST13 in gastric polyps and gastric cancer
}

\author{
Ji Chen, Mingxu Jia* \\ Department of Gastroenterology, Inner Mongolia Medical Baogang Hospital, Baotou, China
}

Received: May 26, 2017

DOI: $10.14725 /$ dcc.v4n3p4

\author{
Accepted: July 8, 2017 \\ Online Published: September 10, 2017 \\ URL: http://dx.doi.org/10.14725/dcc.v4n3p4
}

\begin{abstract}
Objective: To explore the expressions and relationship of HSP70, Apaf-1 and ST13 in the normal gastric mucosa, gastric polyps and gastric cancer, and to provide research basis and a new way for gastric cancer in the early diagnosis and clinical treatment. Methods: Gastric mucosa samples of 72 patients who suffered from surgery in the Inner Mongolia Medical Baogang Hospital by gastroscopy from December 2014 to September 2015 were selected, and divided into normal gastric mucosa group, proliferative polyps group, adenomatous polyps group and adenocarcinoma cancer group. The levels of HSP70, Apaf-1 and ST13 proteins were detected by the Western blotting.

Results: The average IOD of HSP70 expressions was $0.33 \pm 0.05,0.46 \pm 0.05,0.77 \pm 0.07,0.93 \pm 0.04$ respectively in normal gastric mucosa group, proliferative polyps group, adenomatous polyps group and adenocarcinoma cancer group, the difference was statistically significant among four groups $(p<.05)$. The average IOD of Apaf-1 expressions was $0.74 \pm 0.03,0.65 \pm 0.03$, $0.49 \pm 0.06,0.28 \pm 0.05$ respectively, the difference was statistically significant among four groups except for normal gastric mucosa and proliferative polyps $(p<.05)$. The average IOD of ST13 expressions was $0.85 \pm 0.11,0.64 \pm 0.07,0.31 \pm 0.06$, $0.14 \pm 0.01$ respectively, the difference was statistically significant among four groups $(p<.05)$. The expressions of HSP70 and Apaf-1 proteins were negatively correlated in the adenomatous polyps $(r=-0.817, p<.05)$ and adenocarcinoma cancer $(r$ $=-0.816, p<.05)$. The expressions of HSP70 and ST13 proteins were negatively correlated in the adenomatous polyps $(r=$ $-0.818, p<.05)$ and adenocarcinoma cancer $(r=-0.969, p<.05)$. The expressions of Apaf-1 and ST13 proteins were positively correlated in the adenomatous polyps $(r=0.830, p<.05)$ and adenocarcinoma cancer $(r=0.865, p<.05)$.

Conclusions: The expression of HSP70 increased progressively in the process of polyps canceration. It can be regarded as one of the indicators of early diagnosis of gastric cancer. The expressions of Apaf-1 and ST13 decreased progressively in the process of cancelation. The detection of the changes of Apaf-1 and decrease of ST13 specificity in gastric cancer can be learned deeply as diagnostic indicators for gastric cancer. During the canceration of gastric polyps, the expressions of HSP70 and Apaf-1, HSP70 and ST13 proteins were negatively correlated; Apaf-1 and ST13 protein were positively correlated. The joint detection of HSP70, Apaf-1 and ST13 may be helpful in early diagnosis of gastric cancer.
\end{abstract}

Key Words: HSP70, Apaf-1, ST13, Gastric polyps, Gastric cancer

Gastric cancer is one of the most common cancers worldwide. According to the latest data released by the World Health Organization International Agency for Research on Cancer (IARC), about 723,000 people died of gastric car- cinoma worldwide in 2012, ranking the third in the rate of malignant tumors. There are about 325,000 death cases of gastric cancer in China, accounting for $44.9 \%$ of the world mortality rate of gastric cancer. ${ }^{[1]}$ More than $90 \%$ people

\footnotetext{
*Correspondence: Mingxu Jia; E-mail: jiamx2005@163.com; Address: Department of Gastroenterology, Inner Mongolia Medical Baogang Hospital, Baotou, China.
} 
survived within 5-year after treatment for early gastric cancer. ${ }^{[2]}$ Gastroscopy is the gold standard for the diagnosis of gastric diseases, which is easy to be operated and with little damage. At present, gastric polyps are defined as precancerous status of gastric carcinoma. The incidence of gastric polyps is $2 \%-4 \%$ in the population. ${ }^{[3]}$ According to different histopathologies, it can be divided into inflammatory polyps, hyperplastic polyps, adenomatous polyps, fundic gland polyps and so on. Among them, inflammatory polyps are also called pseudo-polyps, accounting for about $80 \%$, and the canceration rate is low. When the diameter of gastric hyperplastic polyps is $>1 \mathrm{~cm}$, the canceration rate will reach $1 \% .{ }^{[4]}$ The detection rate of adenomatous polyps is low but the canceration rate is high, ranging from $6 \%$ to $47 \%,{ }^{[5]}$ and it is also easy to relapse. After follow-up, it is found that the recurrence rate of the patients could reach $2.6 \%$, and $1.3 \%$ of the patients are progressing to gastric cancer.

At present, endoscopic resection is the first choice for the treatment of gastric polyps and a direct way to prevent its canceration. The apoptosis state of cells and its abnormal state on controlling genes may be involved in the occurrence and progression of tumor. The expression of heat-shock protein 70 (HSP70) is regulated by cell cycle. Mutation in tumor cells or existence of abnormal protein stimulates the synthesis of HSP70. It is one of the important influencing factors for the formation and progression of gastric cancer to promote the proliferation of tumor cells or inhibit the apoptosis of cells to present sustained high induced expression. Apoptotic protease activating factor-1 (Apaf-1) is considered to be the true core of apoptosis body and playing a central role in mitochondrial apoptosis pathway. A series of signal transduction cascades reaction in human body are targeted at Apaf-1 to regulate apoptosis body, which is an important tumor suppressor gene. Suppression of tumorigenecity 13 (ST13) was named by the International Nomenclature Committee. The study of ST13 also mainly focused on interreaction of molecular structure and molecular chaperones, which plays a very important role in protein regulation. It may be related to the growth and metastasis of tumor cells. The purpose of this study is to determine the expression level of HSP70 protein, Apaf-1 protein and ST13 protein in gastric polyps and gastric cancer, explore the potential influencing factors in the process of gastric polyp carcinogenesis, and provide clinical basis for the early diagnosis and treatment of gastric cancer.

\section{Materials and methods}

\subsection{General materials}

The gastric mucosa tissues and gastric polyps tissues were collected from patients who visited and underwent gastroscopy at Department of Gastroenterology of Baogang

Published by New Century Science Press
Hospital, Inner Mongolia from December 2014 to September 2015. Through the diagnosis of endoscopy and pathology, normal gastric mucosa group, proliferative polyp group, adenomatous polyp group and gastric cancer group were selected totally for 72 cases. The origin of tissue specimens should also exclude the following cases: $\mathrm{C}^{14}$ breath test suggested helicobacter pylori positive; suffered from familial adenomatous nature polyps before; suffered from malignant neoplasms or combined with malignant neoplasms of other organ systems before; with other endocrine diseases; used NSAIDs, proton pump inhibitor (PPI) or glucocorticoid, etc. for a long time and in recent 4 weeks; combined with chronic organ failure in other systems; polyp sample diameter $>2 \mathrm{~cm}$.

\subsection{Groups}

The experiment samples were divided into 4 groups. Group A: 20 cases of normal gastric mucosa, 12 males and 8 females, aged 27-65 years. All patients performed with abdominal distension and middle upper abdominal discomfort as the main symptom, and no abnormality was found through gastroscopy. Group B: 18 cases for proliferative polyp group, 12 males and 6 females, aged 38-68 years. Proliferative nature polyp was proved through histopathology. Group C: 14 cases of adenomatous polyp group, 6 males and 8 females, aged 46-63 years. Adenomatous nature polyp was proved by endoscopic morphology and histopathology. Group D: 20 cases of adenocarcinoma cancer, 14 males and 6 females, aged 50-95 years. The samples were proved to be adenocarcinoma cancer through endoscopic morphology and histopathology underwent gastroscopy biopsy in Endoscope Center of the Department of Digestive Medicine. Rabbit anti-human HSP70 antibody (US Abcam Company), ST13 antibody (US Abcam Company), Apaf-1 antibody (US Abcam Company), $\beta$-actin antibody (Biyuntian Biotechnology Institute), second antibody (Santa cruz Company), protein marker (Beijing Quanshijin Biotechnology Co., Ltd.).

\subsection{Methods}

\subsubsection{Preparation of stacking gel and separating gel (SDS-PAGE, 1.5 mm thick double-sided)}

After cleaning device and installation, 8\% separating gel and 5\% stacking gel were selected according to the molecular weight of protein to be detected. Configured the gel, and wrapped it with a fresh film after solidification, $4^{\circ} \mathrm{C}$ for the whole night. 


\subsubsection{Extraction of total protein from tissue}

Grinded the shredded tissue in liquid nitrogen and put it into centrifuge tube. Put 150-200 $\mu$ l cracking mixture into each $20 \mathrm{mg}$ of tissues (RIPA:PMSF $=100: 1$ ), and cracked it on the ice box, then centrifuged it at $4^{\circ} \mathrm{C}, 14,000 \mathrm{r} / \mathrm{min}$ for 40 minutes. Measured the protein concentration in the supernatant solution. $150 \mu \mathrm{l}$ sample solution was collected and added with the $50 \mu \mathrm{l}$ of $4 \times$ sample buffer solution, boiled for 5 minutes, cooled and then loaded the sample.

\subsubsection{Electrophoresis}

After installation, electrophoretic buffer $(1 \times$ Running buffer) was poured. $20 \mu \mathrm{l}$ of sample and $5 \mu \mathrm{l}$ of Marker were added for each hole. Firstly set the voltage $50 \mathrm{~V}$ for 30 minutes. When bromophenol blue was about to enter the separating gel, switched the voltage to $100 \mathrm{~V}$ for $90 \mathrm{~min}$, and shut off the power immediately when the bromophenol blue front was about to swim out of the gel.

\subsubsection{Trarsmembran}

Flushed the gel with ultrapure water, then compared the Maker and cut gels with destination bands. Put the gel into the membrane transfer buffer $(1 \times$ Transfer buffer $)$ for immersion equilibrium for 30 minutes. Handled PVDF membrane. The assembly was carried out in the following order according to the requirements of the transfer device: negative electrode - sponge pad - filter paper - gel - PVDF membrane - filter paper - sponge pad - positive electrode, adjusting voltage to $26 \mathrm{~V}$ for 30 minutes.

\subsubsection{Seal}

Took out the PVDF membrane, and washed it in ultrapure water in plate for 5 minutes. Then put it into blocking buffer in $37^{\circ} \mathrm{C}$ water bath and shaked slowly for $2 \mathrm{~h}$.

\subsubsection{Primary antibody incubation}

Rinsed the PVDF membrane for 5 minutes, then diluted HSP70 antibody with $2 \%$ skim milk powder with dilution ratio of 1:5,000, Apaf-1 antibody with dilution ratio of $1: 2,000$, ST13 antibody with dilution ratio of 1:2,500. Fully exposed the diluted first antibody with the PVDF membrane in $4^{\circ} \mathrm{C}$ condition and shook slowly for a night.

\subsubsection{Secondary antibody incubation}

Washed the PVDF membrane with TBST buffer for 3 times, $5 \mathrm{~min} /$ per time, and diluted the second antibody with $5 \%$ skim milk powder. The dilution ratio was 1:5,000. Incubated it at room temperature for $2 \mathrm{~h}$.

\subsubsection{ECL display}

Washed the PVDF membrane with TBST buffer for 3 times, $5 \mathrm{~min} /$ per time. Mixed A solution and B solution $(1: 1)$ of ECL chromogenic solution in light avoiding condition. Dropped the mixed liquid on the film and put it at room temperature for 3 minutes. Pressed it in the dark room, and immediately washed with tap water, fixed, fished, dried and scanned when the strip was found.

\subsubsection{Identification}

After scanning film, IOD value was quantitatively analyzed with image $\mathbf{J}$ software, and the relative expression level of gray value of target protein/gray value of internal reference protein was performed.

\subsection{Statistical treatment}

Statistical analysis was carried out by SPSS 22.0. Statistical data were expressed by $\bar{x} \pm \mathrm{s}$. SNK test was used in comparison among groups. Pearson correlation analysis was used in correlation of HSP70, Apaf-1 and ST13, $p<.05$ showed the difference was statistically significant.

\section{Results}

\subsection{Expression of HSP70 protein in 4 groups}

The IOD value of HSP70 protein/IOD value of internal reference protein in Group A, Group B, Group C, Group D was respectively $0.33 \pm 0.05,0.46 \pm 0.05,0.77 \pm 0.07,0.93$ \pm 0.04 , and the expression of which increased gradually. Through pairwise comparison of four groups, the difference was statistically significant $(p<.05$, see Figure 1$)$.

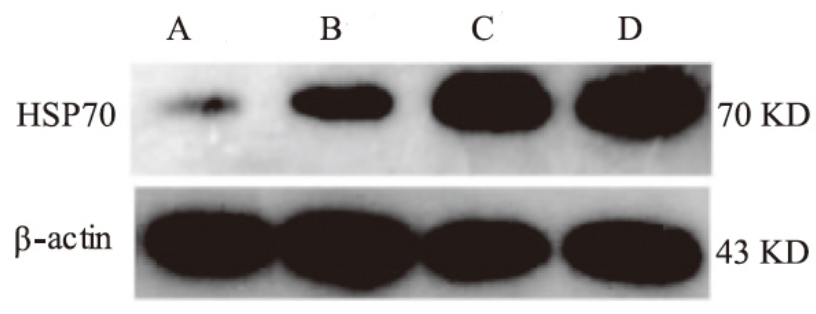

Figure 1: The level of HSP70 protein detected by Western blotting 


\subsection{Expression of Apaf-1 protein in 4 groups}

The average IOD value of Apaf-1 protein in Group A, Group B, Group C, Group D was $0.74 \pm 0.03,0.65 \pm 0.03,0.49$ $\pm 0.06,0.28 \pm 0.05$ respectively, and the expression was gradually decreasing. There was no statistical significant in the difference between Group A and Group B $(p>.05)$. Through pairwise comparison of other groups, the differences were statistically significant ( $p<.05$, see Figure 2 ).

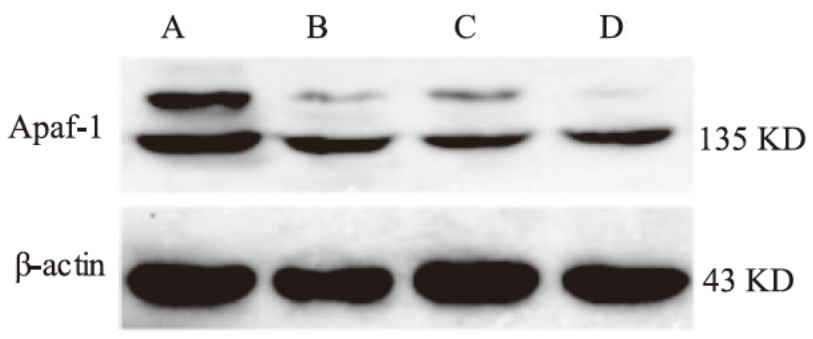

Figure 2: The level of Apaf-1 protein detected by Western blotting

\subsection{Expression of ST13 protein in 4 groups}

The average IOD value of ST13 protein in Group A, Group B, Group C, Group D was $0.85 \pm 0.11,0.64 \pm 0.07,0.31$ $\pm 0.06,0.14 \pm 0.01$ respectively and the expression was gradually decreasing. Through pairwise comparison of four groups, the differences were statistically significant $(p<.05$, see Figure 3).

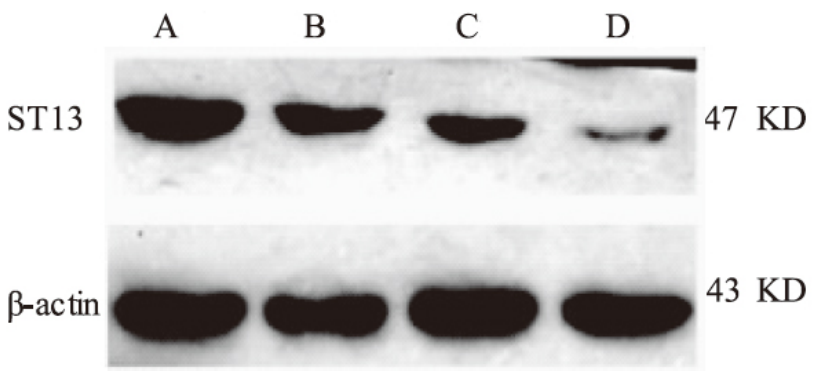

Figure 3: The level of ST13 protein detected by Western blotting

\subsection{Correlation between HSP70 and Apaf-1}

The correlation between HSP70 and Apaf-1 was detected in adenomatous polyp group and gastric adenocarcinoma group by Person method. In adenomatous polyp group, the expressions of HSP70 and Apaf-1 were with negative correlation $(r=-0.817, p<.05)$; in gastric adenocarcinoma group, the expressions of HSP70 and Apaf-1 were with negative correlation $(r=-0.816, p<.05)$.

Published by New Century Science Press

\subsection{The correlation between HSP70 and ST13}

The correlation between HSP70 and ST13 was detected in adenomatous polyp group and gastric adenocarcinoma group by Person method. In adenomatous polyp group, the expressions of HSP70 and ST13 were with negative correlation $(r=-0.818, p<.05)$; in gastric adenocarcinoma group, the expressions of HSP70 and ST13 were with negative correlation $(r=-0.969, p<.05)$.

\subsection{The correlation between Apaf-1 and ST13}

The correlation between Apaf-1 and ST13 was detected in adenomatous polyp group and gastric adenocarcinoma group by Person method. In adenomatous polyp group, the expressions of Apaf-1 and ST13 were with positive correlation $(r=0.830, p<.05)$; in gastric adenocarcinoma group, the expressions of Apaf-1 and ST13 are with positive correlation $(r=0.865, p<.05)$.

\section{Discussion}

With the development and improvement of digestive endoscopy technique, the detection rate of gastric polyp is increasing, and gastric polyp as a form of precancerous lesions of gastric cancer has been paid more attention. This study is to explore the correlation of the expressions of HSP70, Apaf-1 and ST13 protein with the related protein expression of gastric adenoma and gastric cancer as the starting point.

HSP is a kind of protein discovered by Ritossa et al. ${ }^{[6]}$ through "heat shock response". It accompanies by protein molecules to transport in cells without changing the structure of target proteins, and is involved in the folding, stretching and assembly of polycomplex which is called "molecular chaperones". HSP70 is an evolutionarily conserved protein with a molecular weight of about $70 \mathrm{KD}$. The protein can be divided into three domains: ATP binding domain (ATP-BD) of hydrolyzed ATP at $\mathrm{N}$ terminal, peptide binding domain (PBD), which can combine new peptides segments and damaged proteins, and $\mathrm{C}$ terminal with highly conservative terminal sequence that affects the number of HSP70 mRNA translation. It can participate in cell cycle, promote cell proliferation, participate in DNA damage \& repair and process \& extract of antigen, and increase the tolerance of cells to autoimmune factor attack. Tumor is an abnormal proliferative group with infinite proliferation and uncoordinated growth in organism. The decrease in gene stability results in the increase of misfolded proteins, which can increase the demand for HSP70 as "molecular chaperones", and HSP70 has biological functions to participate in cell cycle and anti-apoptosis, which can further encourage the infinite proliferation of tumor cells. This study found that the expression of HSP70 protein was increasing in the tissues of normal gastric mucosa, gastric proliferative 
polyps, adenomatous polyps and gastric cancer. The differences were statistically significant. It is consistent with the current research results at home and abroad. ${ }^{[7,8]}$ Adenomatous polyp is a kind of intraepithelial neoplasia. The requirement for protein synthesis is increased, and more "molecular chaperones" are needed. It is further proved that adenomatous polyp is a form of precancerous lesion, which requires timely treatment.

Apaf-1 is a member of nucleotide binding oligomerization domain family (NOD). It is a tumor suppressor gene expressed in cytoplasm, its molecular weight is about $130 \mathrm{KD}$. There are mainly 3 domains: $\mathrm{N}$ terminal is CARD which forms apoptotic complex with Caspase-9; the middle part is nucleotide binding oligomerization domain; and $\mathrm{C}$ terminal is 12-13 WD40 repetitive domain that can combine with cytochromec. ${ }^{[9]}$ It is distributed in human embryos, human tissues and organs and peripheral white blood cells. Apaf-1 can affect embryo development and is related to chemotherapeutic resistance and prognosis, but its most important biological function is to participate in mitochondrial apoptosis pathway. When the body is stimulated by stress and DNA damage, the Cyt $\mathrm{c}$ of mitochondrial intermembrane cavity and the second activator of mitochondrial Caspase will be released into the cytoplasm. Cyt $\mathrm{c}$ can bind with $\mathrm{C}$ terminal of Apaf-1 to form Apaf-1/Cyt c complex to improve the binding force of Apaf-1 and ATP/dATP, and form Apaf-1 oligomeric heptopolymer. ${ }^{[10]}$ It will then expose CARD, activate Caspase-9 and Caspase-3, and activate downstream components to induce apoptosis. ${ }^{[1,12]}$ Occurrence and progression of tumor are associated with cell apoptosis defects. The inhibition of any link in the mitochondrial apoptosis pathway of Apaf-1 can prevent apoptosis from occurring. For example, HSP70 can directly or indirectly block the combining of Apaf-1, Cyt $\mathrm{c}$ and proCaspase-9. Tanase et al. ${ }^{[13]}$ suggested that Apaf-1 may be a good tumor marker as other important apoptotic factors (such as P53). This study found that the expression of Apaf-1 protein was showing a decreasing trend in normal gastric mucosa, proliferative polyps, adenomatous polyps and gastric cancer tissues. These results suggested that the mitochondrial apoptotic pathway involved by Apaf-1 protein might be inhibited to some extent, which made cancer cells unable to apoptosis. This is similar to domestic and foreign research. ${ }^{[14]}$ Inhibition of tumor cell growth is also used by increasing the activity of Apaf-1 expression clinically. ${ }^{[15]}$ Apaf-1 as a target for cancer treatment not only has become a new direction of clinical cancer treatment, but also provides a new idea for the development of antitumor drugs.

ST13 is considered to be a molecular chaperone of HSP70, with a molecular mass of about $130 \mathrm{KD}$. It is with three main domains: $\mathrm{N}$ terminal - participating in homologous polymerization of molecules, tetratricopeptide repeats (TPR) domain which is combining with the ATP enzyme region of HSP70 and GGMP repeat sequence, and Stil domain which may be interacting with the polypeptide binding re- gion of HSP70. The binding of TPR domain of ST13 to ATP enzyme domain of HSP70 can stabilize ADP conformation, enhance affinity between molecular chaperones and polypeptide substrate, and promote HSP70 to play a role in ensuring protein processing. This study found that the protein expression of ST13 protein was decreasing in the tissues of normal gastric mucosa, gastric hyperplasia polyps, adenomatous polyps and gastric carcinoma. The differences were statistically significant. It was confirmed that the expression of ST13 in gastric cancer and gastric polyp group was lower than that in normal gastric mucosa group, and the specific mechanism is not clear. It may be related to excessive consumption when participating in other apoptotic pathway or inhibition of expression of ST13 in gastric cancer tissues. And may also be its own expression defect. Its biological function needs to be discovered and improved.

This research found that the expression of HSP70 and Apaf1 in adenomatous polyps group and adenocarcinoma cancer group was negatively correlated. "Molecular chaperone" HSP70 not only provides conditions for unlimited cell proliferation in protein processing, but also can directly bind to the $\mathrm{N}$ terminal of CARD domain of Apaf-1, prevents Caspase-9 from binding to it, prevents the formation of apoptotic Apaf-1/Caspase-9 complex, and fails to activate Caspase-3. In addition, the complete expression of HSP70 can inhibit the release of Cyt c from mitochondria. Therefore, HSP70 also prevents the formation of Apaf-1/Cyt c complex to some extent. It can be seen that HSP70 can play a role in the release of Cyt $\mathrm{c}$ and the initiatial activation of Caspase, which prevents the activation of mitochondrial apoptosis pathway of Apaf-1, affects the expression of Apaf-1 and apoptosis pathway, and prevents apoptosis of cells. The negative correlation of HSP70 and ST13 in adenomatous polyps group and adenocarcinoma cancer group suggests that there may be a potential correlation between HSP70 and ST13 during the process of tumor formation. Over expression of HSP70 is a prerequisite for the formation of malignant tumors in human tissues and is one of the important influencing factors in the process of occurrence and progression of gastric cancer. The combination of ST13 TPR structure domain and HSP70 can stabilize the ADP conformation of HSP70, which makes HSP70 function better. ST13 as a molecular chaperone plays a stable and positive role in the regulation of HSP70. But in this study, it was found that HSP70 and ST13 were negatively correlated in adenocarcinoma cancer group. It is not consistent with the biological function of molecular chaperone of ST13 and this result is the same as that of our previous study. ${ }^{[16]}$ In the research of carcinoma of colon, Wang et al. ${ }^{[17]}$ found that the expression of ST13 in carcinoma of colon tissues was obviously lower than that in the adjacent normal mucosa tissues, which indicated that the expression of ST13 was defective after canceration. These results suggested that HSP70 might have a direct or indirect down-regulation effect on ST13 of its molecular chaperone in gastric cancer 
tissues. ST13 might also be antagonistic to other oncogenes or tumor suppressor genes in gastric cancer tissue and precancerous lesions. Apaf-1 and ST13 were positively correlated in adenomatous polyps group and adenocarcinoma cancer group. ST13 can mediate the TPR domain of important molecular element of protein interaction. So ST13 can bind to many proteins. The ATP sequences of Apaf-1 and HSP70 have local homology. ST13 may interact directly with the ATP binding domain of Apaf-1. It is inferred that it may be involved in apoptosis controlled by Apaf-1. In gastric cancer tissues, the expression of Apaf- 1 decreases, and then the expression of ST13 may be down-regulated. At present, the research on the expression difference of ST13 in cancer has just started. Many molecular biological functions have not been clarified. We need to further explore the two mechanisms of mutual regulation.

\section{References}

[1] Ferlay J, Soerjomataram I, Dikshit R, et al. Cancer incidence andmortality worldwide: sources, methods and major patterns in GLOBOCAN 2012. Int J Cancer. 2015; 136(5): E359-E386. PMid: 25220842. https://doi.org/10.1002/ijc. 29210

[2] Isobe $\mathrm{Y}$, Nashimoto A, Akazawa $\mathrm{K}$, et al. Gastric cancer treatmemnt in Japan: 2008 annual report of the JGCA nationwide registry. Gastric Cancer. 2011; 14(4): 301-316. PMid: 21894577. https://doi.org/10.1007/s10120-011-0085-6

[3] Ye L, Liu T, Leng AM, et al. Analysis of clinical characteristics and treatment strategy for gastric polyps. China Journal of Modern Medicine. 2011; 21(5): 659-664.

[4] Ahn JY, Son DH, Choi KD, et al. Neoplasms arising in large gastric hyperplastic polyps: endoscopic and pathologic features. Gastrointest Endosc. 2014; 80(6): 1005-1013. PMid: 24929480. https: //doi.org/10.1016/j.gie.2014.04.020

[5] Sun CK, Yang KC, Liao CS. Endoscopic management of gastric polyp with outlet obstruction without polypectomy. Case Rep Gastroenterol. 2011; 5(2): 267-271. PMid: 21887127. https://doi. org/10.1159/000328443

[6] Ritossa FM. A new puffing pattern induced by heat shock and DNP in Drosophilia. Experientia. 1962; 18(12): 571-573. https : //doi.org/10.1007/BF02172188

[7] Lee HW, Lee EH, Kim SH, et al. Heat shock protein 70 (HSP70) expression is associated with poor prognosis in intestinal type gastric cancer. Virchows Arch. 2013; 463(4): 489-495. PMid: 23913168. https://doi.org/10.1007/s00428-013-1461-x

[8] Wu YM, Jv LT, Xie HZ. Expression of heat shock protein 70 in patients with chronic atrophic gastritis. China Journal of Modern Medicine. 2010; 20(2): 179-182.
In a word, HSP70, Apaf-1 and ST13 have obvious abnormal expression in the process of gastric polyp to gastric cancer, and they are correlated with each other. Monitoring HSP70, Apaf-1 and ST13 will help screen out high-risk patients with gastric cancer. Especially, the low expression of ST13 protein in gastric cancer is helpful for the diagnosis of potential gastric cancer. This study detected the expression and expression trend of three kinds of factors in human tissues, which is similar to the results of many in vitro cell culture and animal model experiments. It is helpful to explore the similarities and differences between the basic experimental research and clinical human tissue research. It may also be a potential target for future research on targeted therapy.

\section{Conflicts of Interest Disclosure}

The authors have no conflicts of interest related to this article.

[9] Reubold TF, Wohlgemuth S, Eschenburg S. Crystal structure of full length Apaf-1: how the death signal is relayed in the mitochondrial pathway of apoptosis. Structure. 2011; 19(8): 1074-1083. PMid: 21827944. https://doi.org/10.1016/j.str.2011.05.013

[10] Zhou M, Li Y, Hu Q, et al. Atomic structure of the apoptosome: mechanism of cytochrome $\mathrm{c}$ and dATP-mediated activation of Apaf1. Genes Dev. 2015; 29(22): 2349-2361. PMid: 26543158. https : //doi.org/10.1101/gad.272278.115

[11] Yuan S, Topf M, Reubold TF, et al. Changes in Apaf-1 conformation that drive apoptosome assembly. Biochemistry. 2013; 52(13): 2319-2327. PMid: 23521171. https://doi.org/10. 1021/bi301721g

[12] $\mathrm{Hu} \mathrm{Q}, \mathrm{Wu} \mathrm{D}$, Chen W, et al. Molecular determinants of Caspase9 activation by the Apaf-1 apoptosome. Proc Natl Acad Sci USA. 2014; 111(46): 16254-16261. PMid: 25313070. https://doi. org/10.1073/pnas. 1418000111

[13] Tanase C, Albulescu R, Codrici E, et al. Decreased expression of APAF-1 and increased expression of cathepsin B in invasive pituitary adenoma. Onco Targets Ther. 2015; 8: 81-90. PMid: 25565868.

[14] Yin W, Mo XL, Wei HM, et al. Expression and significance of Survivin, Apaf-1 and Caspase-9 in process of peritoneal metastasis of gastric cancer. Guangdong Medical Journal. 2014; 35(2): 221-223.

[15] Tan W, Lu J, Huang M, et al. Anti-cancer natural products isolated from Chinese medicinal herbs. Chin Med. 2011; 6(1): 1-15. PMid: 21777476. https://doi .org/10.1186/1749-8546-6-27

[16] Chen J, Niu HS. Expression of ST13 and HSP70 in gastric polyps and gastric cancer. World Chin J Digestol. 2013; 21(29): 3059-3066. https://doi.org/10.11569/wcjd.v21.i29.3059

[17] Wang LB, Zheng S, Zhang SZ, et al. Expression of ST13 in colorectal cancer and adjacent normal tissues. World J Gastroenterol. 2005; 11(3): 336-339. PMid: 15637739. https://doi.org/10.3748/ wjg.v11.i3.336 